\title{
Reconstruction of the left subclavian artery in arch repair: An end-to-side anastomosis technique
}

Tomonari Fujimori ${ }^{1}$, Homare Okamura ${ }^{1}$, Yuichiro Kitada ${ }^{1}$, Yohnosuke Wada ${ }^{1}$, and Hideo Adachi $^{1}$

${ }^{1}$ Nerima Hikarigaoka Byoin

February 8, 2022

\begin{abstract}
In total arch replacement, using frozen elephant trunk (FET) or elephant trunk techniques ensures proximalization of the distal anastomosis. However, in some cases, the left subclavian artery (LSCA) is deeply located and difficult to visualize. Therefore, surgeons face technical challenges during the LSCA reconstruction. We report an end-to-side anastomosis technique that enables safer and easier anatomical reconstruction of the LSCA.
\end{abstract}

\section{Title:}

Reconstruction of the left subclavian artery in arch repair: An end-to-side anastomosis technique

Authors:

Tomonari Fujimori, MD, Homare Okamura, MD, PhD, Yuichiro Kitada, MD,

Yohnosuke Wada, MD, Hideo Adachi, MD, PhD

Institutions and affiliations:

Department of Cardiovascular Surgery, Nerima Hikarigaoka Hospital, Tokyo, Japan

\section{Corresponding author:}

Homare Okamura, MD, PhD

2-11-1 Hikarigaoka, Nerima-Ku, Tokyo 179-0072, Japan

TEL: +81-3-3979-3611, FAX: +81-3-3979-3787

E-mail address: homareo@jadecom.jp

\section{Conflict of interest}

All the authors declare no competing interests.

\section{Ethics statement}

IRB approval for case reports of a single patient is not required in our institution.

\section{Informed consent}

The patient provided informed consent for the publication of this report.

\section{ORCID ID}


0000-0003-2142-0701

\begin{abstract}
In total arch replacement, using frozen elephant trunk (FET) or elephant trunk techniques ensures proximalization of the distal anastomosis. However, in some cases, the left subclavian artery (LSCA) is deeply located and difficult to visualize. Therefore, surgeons face technical challenges during the LSCA reconstruction. We report an end-to-side anastomosis technique that enables safer and easier anatomical reconstruction of the LSCA.
\end{abstract}

\title{
Key Words
}

Arch repair * Left subclavian artery* Reconstruction

\section{Introduction}

In total arch replacement, using FET or elephant trunk techniques brings proximalization of the distal anastomosis, from Zone3 to Zone2. However, the method for the reconstruction of the LSCA remains debatable. Herein, we report an end-to-side anastomosis technique, which can be used safely in the reconstruction of the LSCA in arch repair and offers several advantages.

\section{Surgical Technique}

A 70-year-old woman was diagnosed with chronic aortic dissection. Contrast-enhanced computed tomography $(\mathrm{CT})$ demonstrated a type A aortic dissection with a primary intimal tear in the ascending aorta. The aortic diameters were $76 \mathrm{~mm}$ in the ascending aorta and $74 \mathrm{~mm}$ in the descending aorta (Figure 1).

Elective surgery was performed via a median sternotomy. Cardiopulmonary bypass was initiated via cannulation of the ascending aorta, right atrium, and left ventricular vent. Hypothermic circulatory arrest was induced at a rectal temperature of $25^{\circ} \mathrm{C}$. Retrograde and antegrade cold blood cardioplegia were administered intermittently. Antegrade cerebral perfusion was injected into the three supra-aortic vessels. LSCA reconstruction was performed before the distal anastomosis. First, the origin of the LSCA was ligated by silk threads, which were then pulled caudally for better exposure of the LSCA. By clamping the distal LSCA, the anterior wall of the LSCA was incised longitudinally. Subsequently, a 5-cm length graft, which formed a part of the 4-branched Dacron tube graft ( $\mathrm{J}$ graft Japan; Lifeline, Tokyo, Japan), was anastomosed to the LSCA using 5-0 polypropylene sutures (Figure 2-a). The LSCA was declamped, and antegrade cerebral perfusion to the LSCA was ensured through the anastomosed graft (Figure 2-b).

Thereafter, distal anastomosis was performed by first inserting the 5-cm graft as the elephant trunk, and the 4-branched graft was anastomosed to the transected aorta. A proximal anastomosis was performed, and the heart was restarted. The left common carotid and innominate arteries were reconstructed. Finally, the graft anastomosed to the LSCA was reconstructed (Figure 2-c). Postoperative CT showed antegrade flow to the supra-aortic vessels (Figure 2-d).

\section{Discussion}

In arch repair, the LSCA is deeply located, which makes it difficult to visualize and therefore, creates technical challenges during surgical repair in some patients. Therefore, several innovations have been designed to facilitate the reconstruction of the LSCA, including direct anastomosis, carotid-LSCA transposition or bypass, left axial extra-anatomic bypass, and ligation without revascularization [1-2]. Direct end-to-end anastomosis is the simplest. However, anastomosis and hemostasis are technically difficult in patients with deep LSCAs. The LSCA easily retracts cranially following its transection. In contrast, an extra-anatomic bypass of the left axial bypass through the left thorax is used to avoid complex LSA anastomosis; however, it requires an additional incision to expose the left axillary artery. This procedure is time-consuming and is accompanied by the risk of brachial plexus injury and wound infection [3]. Furthermore, postoperative adhesion around the bypass in the left thorax can complicate future open surgery via left thoracotomy for the enlarged descending aorta. 
Our reconstruction technique provides excellent exposure of the LSCA by pulling the origin of the LSAC caudally, which simplifies the anastomosis. Hemostasis after reconstruction is feasible, as the anastomosis in the anterior wall of the LSCA is easily visualized. The disadvantage of our technique is the extension of total circulatory arrest time which requires almost ten minutes.

The selection of patients for this technique is important, and it is contraindicated in patients with dissected LSCA or LSCA with atherosclerotic plaques, which can be repaired by the left axial extra-anatomic bypass or fenestrated FET techniques [4].

\section{Conclusion}

The end-to-side anastomosis technique for LSCA reconstruction in arch repair is a simple alternative; for confirming the safety and efficacy of the technique, more follow-up studies in a larger series would be required.

\section{References}

1. Ho JYK, Bashir M, Jakob H, Wong RHL. Management of left subclavian artery in total arch replacement and frozen elephant trunk procedure. JTCVS Tech. 2021;7:36-40.

2. Hage A, Ginty O, Power A, Dubois L, Dagenais F, Appoo JJ, et al. Management of the difficult left subclavian artery during aortic arch repair. Ann Cardiothorac Surg. 2018;7:414-21.

3. Sabik JF, Nemeh H, Lytle BW, Blackstone EH, Gillinov AM, Rajeswaran J, et al. Cannulation of the axillary artery with a side graft reduces morbidity. Ann Thorac Surg. 2004;77:1315-20.

4. Okamura H, Arakawa M, Takeuchi T, Adachi H. The fenestrated frozen elephant trunk technique for acute type A aortic dissection. J Thorac Cardiovasc Surg. 2018;156:e75-e7.

\section{Figure Legends}

Figure 1. a-c, Preoperative contrast-enhanced computed tomography (CT) image: a,c ) Dissecting aneurysm of ascending aorta and $\mathbf{b}$ ) descending aorta. $\mathbf{d}$ ) Preoperative three-dimensional CT image.

Figure 2. a-c , Intraoperative view: a ) The left subclavian artery (LSCA) was well exposed by ligating and pulling caudally (toward dashed arrow). $\mathbf{b}$ ) The graft was anastomosed to the LSCA by the end-to-side anastomosis technique (solid arrow).c ) Graft-to-graft anastomosis. $\left(^{*}\right) \mathbf{d}$ ) Postoperative three-dimensional computed tomography image.

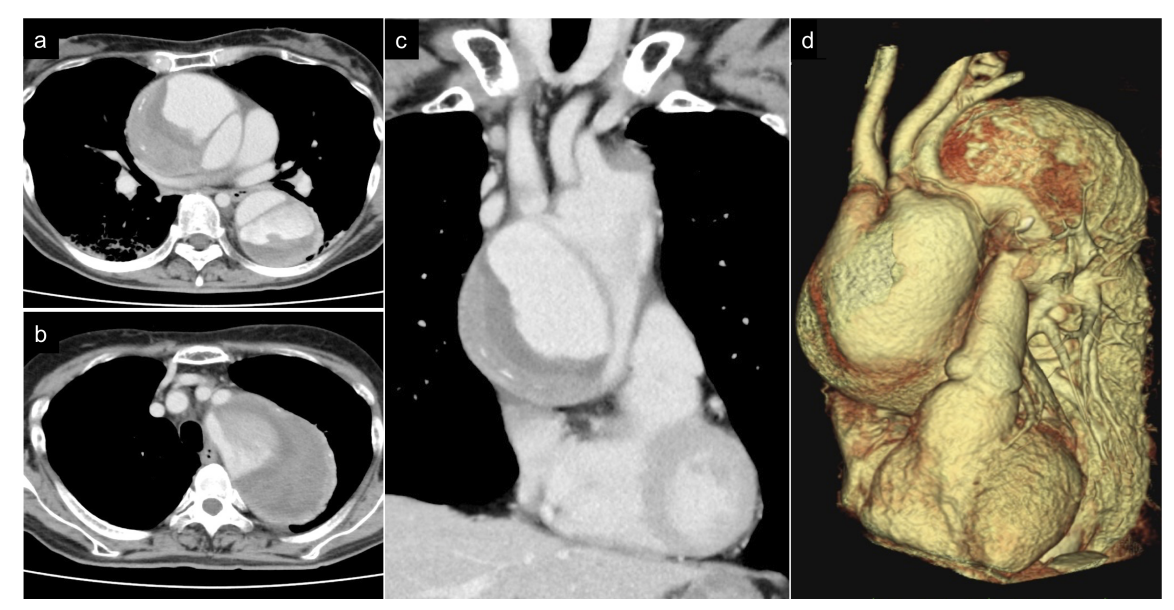




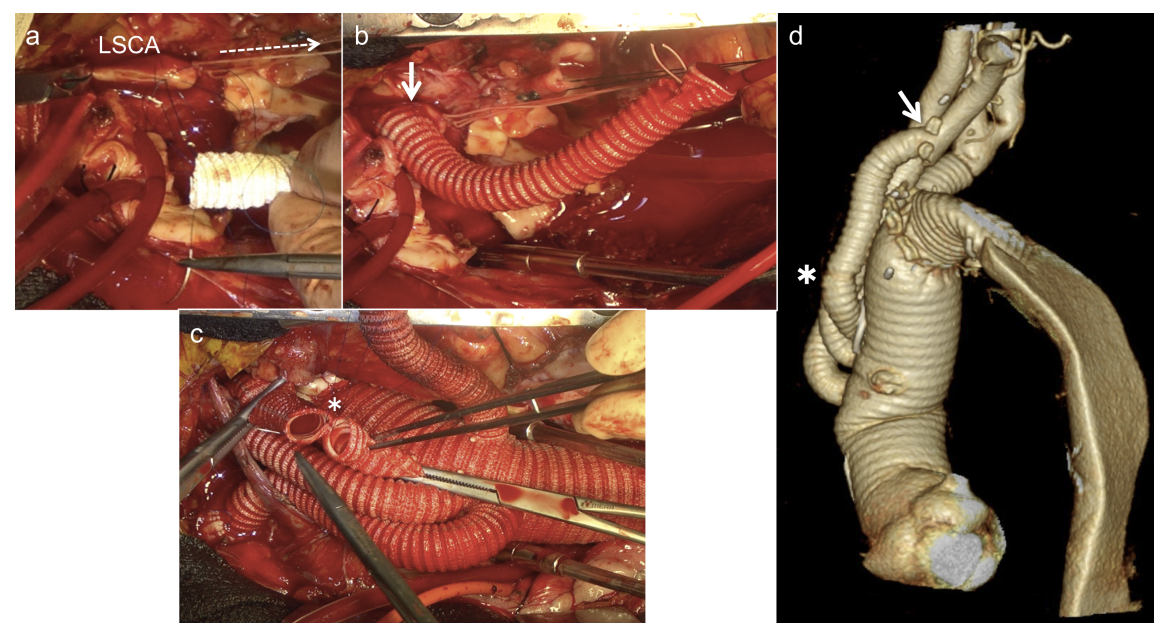

